\title{
METHOD FOR ESTIMATING CLUTTER LIMITED GEOSYNCHRONOUS SYNTHETIC APERTURE RADAR PERFORMANCE
}

\author{
C. Convenevole and S.E. Hobbs School of Aerospace, Transport and Manufacturing \\ Cranfield University \\ Cranfield, United Kingdom \\ c.convenevole@cranfield.ac.uk
}

\begin{abstract}
This paper reviews the Geosynchronous Synthetic Aperture Radar (GeoSAR) literature and highlights a gap in land clutter theory. The gap concerns clutter models with weather dependence for incidence angles between 20 and 70 degrees. We update with an improved clutter model the System Performance method presented at IGARSS 2016 to estimate the SAR system performance taking into account the weather statistics and the landcover of the target area. The performance statistics obtained with this method allow to estimate the fraction of time (e.g. percentage of a month) in which the SAR system can accomplish the user's requirement. So far the method has been developed for short vegetation (specifically wheat), as source of clutter, and for bare land and urban area as other target areas. A full clutter model is still under development, but results are presented for the weather dependence of the coherent fraction of the scattered power. Since the azimuth spread of clutter power for GeoSAR can exceed the beam footprint in strong weather conditions, we expect improved image quality in these conditions. Two example of the effect of different clutter power Probability Density Function are briefly presented.
\end{abstract}

Index Terms-GeoSAR, Geosynchronous SAR, SAR Performance Estimation Method

\section{INTRODUCTION}

The idea of a Geosynchronous SAR (GeoSAR) is not new [1] and many types of mission have been proposed in the last twenty years: some monostatic [2]-[5], other bistatic [6]-[8] and even multistatic [9]. Despite all these studies, no mission has flown yet or has even gone further than the phase-0. Many aspects have been investigated, such as the atmospheric corruption of the image [10], its possible compensation [11] and the interferometry applications [7]. But there is still uncertainty about the performance achievable on non-static target areas. Such uncertainty could be particularly important for those mission concepts [3]-[5], [7]-[9] that are characterised by a low azimuth speed, and which use integration time from minutes to hours.

On the opposite, conventional Low Earth Orbit SAR (LEOSAR) have a short integration time. Thus, a moving target with a velocity component along the slant range is focused in a displaced position. This phenomenon is called azimuth shift. In the GeoSAR concepts we cited, the azimuth shift can cause effects more difficult to be predicted: when the target scene is not-static, the long integration time causes the moving target to have different velocities during the integration time. Thus the target is not simply displaced, but it is smeared in the azimuth direction. Moreover, the low azimuth speed, coupled with the long slant range (that is a way longer than in LEOSAR), causes this displacement to be a way bigger than in conventional LEO-SAR. This displacement could be so large that the clutter is pushed even outside the beam footprint.

In order to estimate the performance achievable in realistic weather conditions on real landscapes by these low azimuth speed missions, a method has been developed [12]. During the development of this method, the review of the available literature [13]-[15] has shown the need for a new clutter model, due to the different system geometric conditions. Indeed, the Billingsley model has been developed for groundbased radar to model trees windblown clutter and even if it has been extended to other landcovers, the model is valid for a grazing angle that is less than $10^{\circ}$. Conversely, in the GeoSAR mission concepts we cited, the incidence angle is typically between $20^{\circ}$ and $70^{\circ}$.

Moreover, the available clutter models [13]-[15] use an empirical approach aiming at fitting the clutter signal. Whereas, we built a physics-based clutter model that takes in account the target movement and has a wider range of application: in fact it can be applied to every incidence angle condition.

This new clutter model has been developed for wheat (representing short vegetation) in order to demonstrate the method capabilities. In section II we provide a description of the method; in section III we present the system geometry; in section IV we define the Signal to Clutter Ratio and the Signal to Disturbance Ratio; in section V we describe the submodels that are part of the method and in section VI we discuss the simulation results and outline the future work needed to complete the study.

\section{Method DEscRiption}

The method outlined in [12] has been updated in order to use the wheat clutter model that we developed. As we can see in Fig. 1 on the left, the Performance Estimation Method takes as input the landscape statistics, the weather statistics, the space system parameters and the user requirements to estimate the performance statistics. This means to quantify for what fraction of the time the user requirements are satisfied by the system performance during different times of year, having 


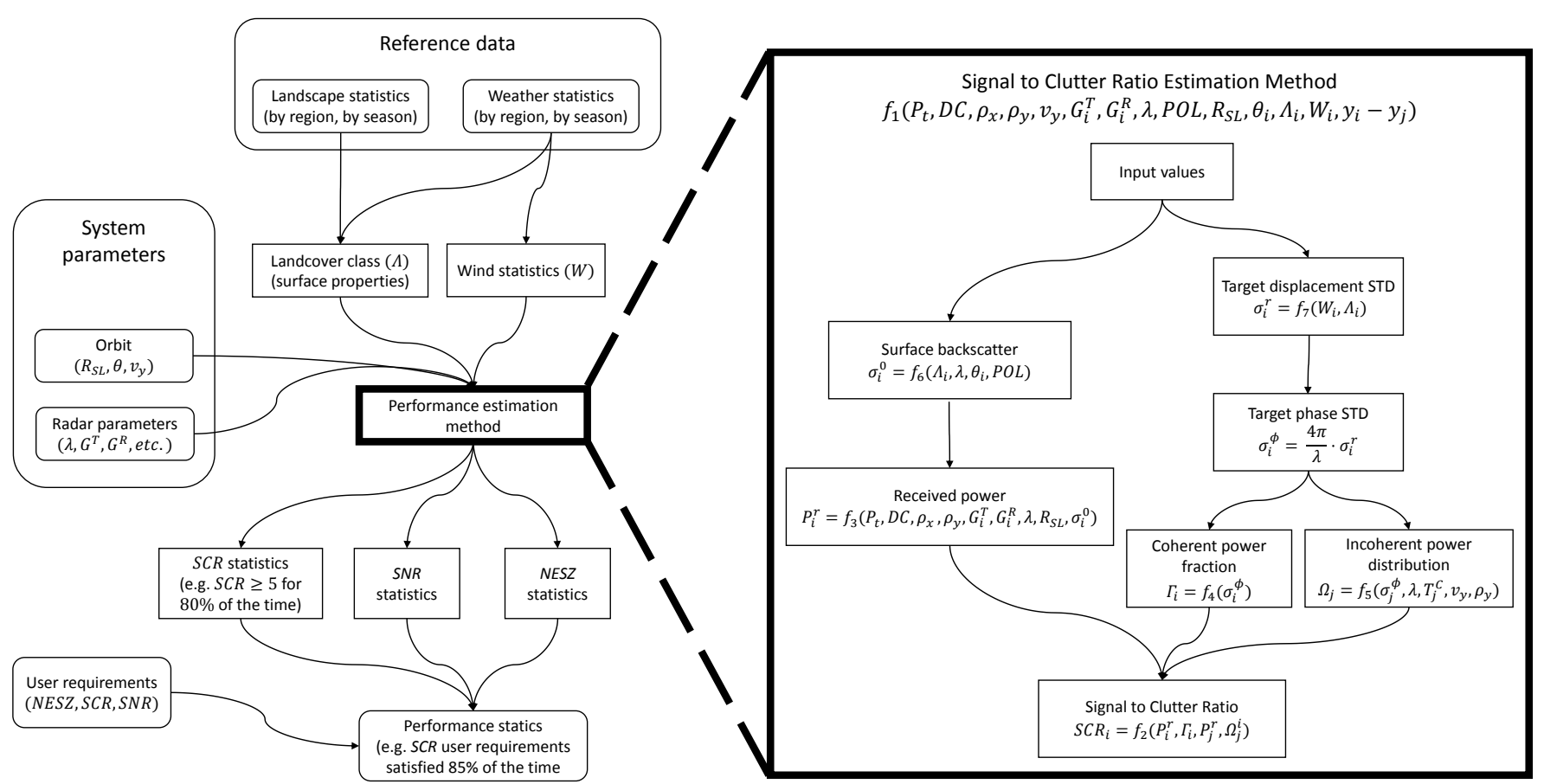

Fig. 1: On the left, the Performance Estimation Method diagram. On the right inside the box, the Signal to Clutter Ratio Estimation Method.

considered the effects of different target locations and different landscapes.

Depending on of which final product we want to estimate the performance, we have to choose the appropriate function model to be used for computing the performance statistics. These function models are slightly different but the basic idea is simulating the image using the target area statistics (landcover and weather). In this way, we estimate the final product and its performance statistics in realistic conditions. For example, we have a different function model for the Signal to Clutter Ratio $(S C R)$, for the Signal to Noise Ratio $(S N R)$ and for the Noise Equivalent Sigma Zero (NESZ).

We can see an example in the right part of Fig. 1 where the sub-method to compute the Signal to Clutter Ratio statistics is represented inside the box. We can represent this submethod as a function ( $f_{1}$ in the diagram): it takes as input the integration time $\left(T_{\text {int }}\right)$, the transmitted power $\left(P_{t}\right)$, the Duty Cycle $(D C)$, the pulse repetition frequency $(P R F)$, the range resolution $\left(\rho_{x}\right)$, the azimuth resolution $\left(\rho_{y}\right)$ and the azimuth velocity $\left(v_{y}\right)$, the transmitter and receiver antenna gain $\left(G^{T}, G^{R}\right)$, the wavelength $(\lambda)$, the antenna polarisation (POL), the target slant range $\left(R_{S L}\right)$, the incidence angle $(\theta)$ and the landcover $(\Lambda)$.

For example, the landscape statistics describe the landcover classes in the target scene. The landcover together with the polarization of the transmitter and receiver antennas, wavelength and incidence angle, determine the backscatter coefficient $\left(\sigma^{0}\right)$.

The weather statistics include the wind statistics and the season. The season together with the landcover allows to take in account the plant growth and therefore to choose

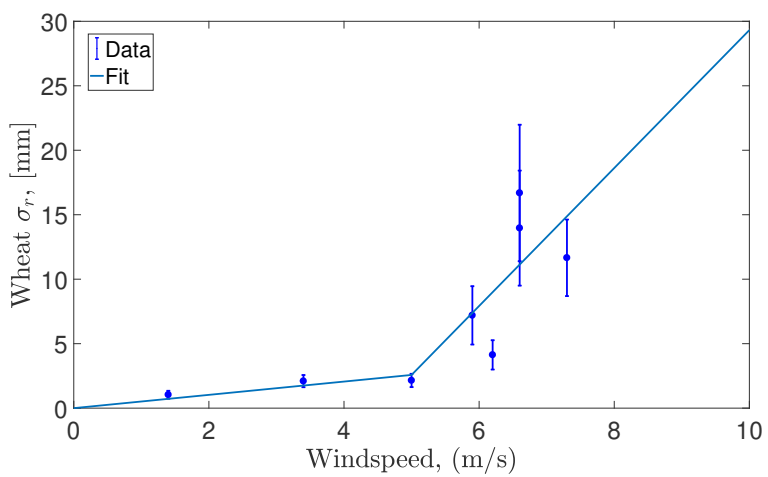

Fig. 2: Two line fit of the displacement standard deviation as function of the windspeed, based on wheat movement database [16]; landcover: wheat; season: summer.

the appropriate function to compute the target displacement standard deviation $\left(\sigma^{r}\right)$. For example in Fig. 2 we can see the data of the wheat movement standard deviation during the summer and the corresponding fit with two lines.

We model the target displacement because we chose to build a physics based clutter model and not a model that just fits the clutter signal as done before [13]-[15].

\section{System GEOMETRY}

Figure 3 shows the system geometry. It is worth noting that the annulus of constant range from the satellite is centred around the sub-satellite point, which is not always on the equator. The position on the annulus is identified by one 


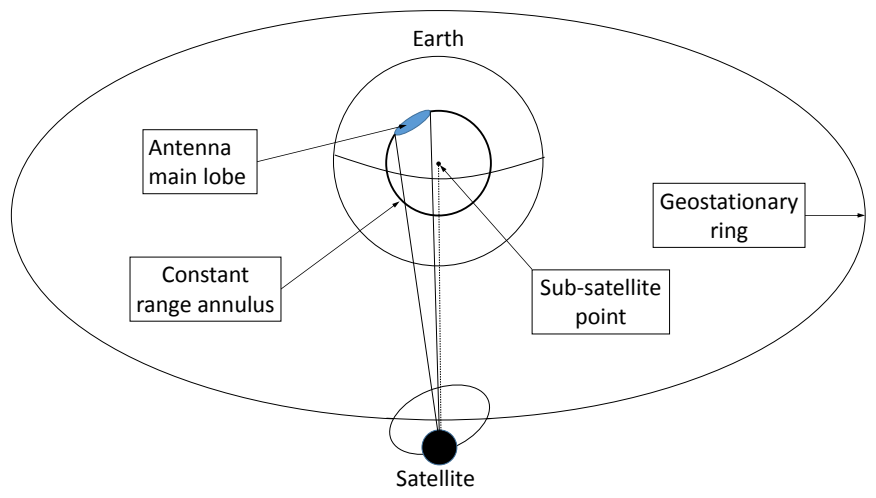

Fig. 3: System geometry (not in scale) for the concepts [3], [5]. The satellite relative orbit around the geostationary position is exaggerated.

coordinate. The annulus itself is identified by the slant range. If the inclination is low and the eccentricity is small like in the some mission concepts [3], [5], we can represent the satellite motion with its orbit around a geostationary position.

\section{Signal to Disturbance Ratio Definition}

The signal received for a single pulse is defined by the following equation:

$$
P^{r}=\frac{P_{t} G^{T} A_{r} \sigma}{(4 \pi)^{2} R^{4}}
$$

as shown in [17, Eq.1.1], where we have the receiver effective area $\left(A_{r}\right)$, the radar cross section $(\sigma)$, the range $(R)$. The radar cross section $\sigma$ can be expressed as

$$
\sigma=\sigma^{0} \rho_{x} \rho_{y} \cos (\theta),
$$

and we can substitute the antenna gain (assuming the system monostatic, thus $G=G^{R}=G^{T}$ )

$$
G=\frac{4 \pi A_{r}}{\lambda^{2}} .
$$

In this way we get the power received (for both signal and clutter) for a single pulse:

$$
P^{r}=\frac{P_{t} G^{2} \lambda^{2} \rho_{x} \rho_{y} \sigma^{0} \cos (\theta)}{(4 \pi)^{3} R^{4}}
$$

The receiver system thermal noise (plus other noise sources with similar distribution) can be expressed as

$$
P_{n}=k T_{n} B_{n}
$$

where $T_{n}$ is a semi-fictitious noise temperature as stated by [17, Eq.2.31].

In order to have a uniform notation we can introduce the noise figure $\left(F_{n}\right)$, and we get

$$
B_{n}=B F_{n} .
$$

We define the Signal to Disturbance Ration $(S / D)$ as

$$
S / D_{i}=\frac{S_{i}}{N+C}=\frac{S_{i}}{N+\sum_{j \neq i} C_{j}^{i}}
$$

where we have the signal power $(S)$, the noise power $(N)$ and the clutter power $(C)$. The subscript $j$ and the superscript $i$ on the clutter denotes that the clutter comes from the pixel $j$ and it is focused on the pixel $i$. Thus, for a single pulse we get

$$
S / D_{i}=\frac{\frac{P_{t} G^{2} \lambda^{2} \rho_{x} \rho_{y} \sigma_{i}^{0} \cos (\theta)}{(4 \pi)^{3} R^{4}}}{\left[k T_{0} B F_{n}\right]+\sum_{j \neq i} \frac{P_{t} G^{2} \lambda^{2} \rho_{x} \rho_{y} \sigma_{j}^{0} \cos (\theta)}{(4 \pi)^{3} R^{4}}}
$$

If we consider the pulse compression gain $\left(G_{p c}\right)$ and the coherent integration $\left(G_{i n t}\right)$ of the signal we get an improvement factor

$$
\text { Improvement factor }=G_{p c} G_{i n t}=\frac{\tau_{i}}{\tau_{0}} \frac{P R F \cdot L}{v}
$$

as shown in [17, Eq.21.51], where we have the uncompressed pulse length $\left(\tau_{i}\right)$, the compressed pulse length $\left(\tau_{0}\right)$ and the integration time $\left(T_{\text {int }}=L / v\right)$ expressed as the ration the length of the synthetic antenna $(L)$ and the radar velocity $(v)$.

For the clutter signal we have two improvements: the pulse compression and the (partially) integration gain. The integration of the clutter is partially coherent, thus

$$
G_{i n t}^{c l}=n_{c} \sqrt{n_{i n c}},
$$

where we have the number of pulses in which the clutter is coherent $\left(n_{c}\right)$ and the number of pulses in which the target is incoherent $\left(n_{\text {inc }}\right)$. The number of clutter coherent pulses is given by

$$
n_{c}=P R F \cdot \tau^{c}
$$

where we have the clutter coherence time $\left(\tau^{c}\right)$, that is the time for which the clutter remains coherent. Combining the 10 and the 11 , we get

$$
G_{i n t}^{c l}=n_{c} \sqrt{\frac{P R F \cdot T_{i n t}}{n_{c}}}=\sqrt{P R F \cdot T_{i n t} \cdot n_{c}}
$$

that leads to

$$
G_{i n t}^{c l}=P R F \sqrt{T_{i n t} \cdot \tau^{c}}
$$

So we get the following expression for the Signal to Disturbance Ratio of a focused image

$$
S / D_{i}=\frac{G_{p c} P R F \cdot T_{i n t} \cdot \frac{P_{t} G^{2} \lambda^{2} \rho_{x} \rho_{y} \sigma_{i}^{0} \cos \left(\theta_{i}\right)}{(4 \pi)^{3} R^{4}}}{\left[k T_{0} B F_{n}\right]+\sum_{j \neq i}\left[G_{p c} G_{i n t}^{c l} \frac{P_{t} G^{2} \lambda^{2} \rho_{x} \rho_{y} \sigma_{j}^{0} \cos \left(\theta_{j}\right)}{(4 \pi)^{3} R^{4}}\right]}
$$


where we highlighted that the signal comes from the cell $i$ and the clutter is the sum of the clutter components coming from all the other cell $j$ in the same range gate.

We can rewrite it as

$$
S / D_{i}=\frac{\frac{\tau_{i}}{\tau_{0}} P R F \cdot T_{i n t} \cdot P_{i}^{r} \Gamma_{i}}{\left[k T_{0} B F_{n}\right]+\sum_{j \neq i} G_{p c} G_{i n t}^{c l} P_{j}^{r} \Omega_{j}^{i}}
$$

where we introduced the coherent signal fraction $\left(\Gamma_{i}\right)$ that comes from the pixel itself, and the incoherent signal fraction $\left(\Omega_{j}^{i}\right)$ backscattered (due to the focusing process) from the cell $j$ on the cell $i$.

$$
\begin{aligned}
& \frac{P_{i}^{s i g}}{P_{i}^{r}}=\Gamma_{i} \\
& \frac{P_{i}^{c l}}{P_{i}^{r}}=\Omega_{i}
\end{aligned}
$$

And $\Omega_{i}$ is the total fraction of the signal, effectively the total clutter, backscattered from the cell $i$ to somewhere else.

We can substitute the Duty Cycle [17, eq. 21.57]

$$
P_{a v}=P_{t} \tau_{i} P R F=P_{t} D C
$$

and then note that usually $\mathrm{B}$ is designed to be the reciprocal of $\tau_{0}$ [17, eq. 21.58]

$$
B \tau_{0}=1
$$

and we get

$$
S / D_{i}=\frac{D C \cdot T_{i n t} \cdot P_{i}^{r} \Gamma_{i}}{\left[k T_{0} F_{n}\right]+\sum_{j \neq i}\left[D C \sqrt{\left(T_{i n t} \tau_{j}^{c}\right)} P_{j}^{r} \Omega_{j}^{i}\right]}
$$
ing:

We can highlight the functional dependences in the follow-

$$
\begin{gathered}
P_{i}^{r}=f_{3}\left(P_{t}, D C, \rho_{x}, \rho_{y}, G_{i}^{T}, G_{i}^{R}, \lambda, R_{S L}, \sigma_{i}^{0}\right) \\
\Gamma_{i}=f_{4}\left(\sigma_{i}^{\phi}\right) \\
\Omega_{j}=f_{5}\left(\sigma_{j}^{\phi}, \lambda, T_{j}^{C}, v_{y}, \rho_{y}, y_{i}-y_{j}\right)
\end{gathered}
$$

All the variables are time-dependent and can vary during the integration time. In the most general case we can allow every variable to change during the image integration time but we assume that most of the properties do not vary during the integration time. The only variable that we consider could vary during the integration time is the mean windspeed. The method can be updated to include the time variation of the properties.

SCR here is statistical, so it implies a certain averaging time such that weather and crop state has not changed significantly. The clutter is a multiplicative noise, so if we increase the transmitted power, we also increase the received clutter power.

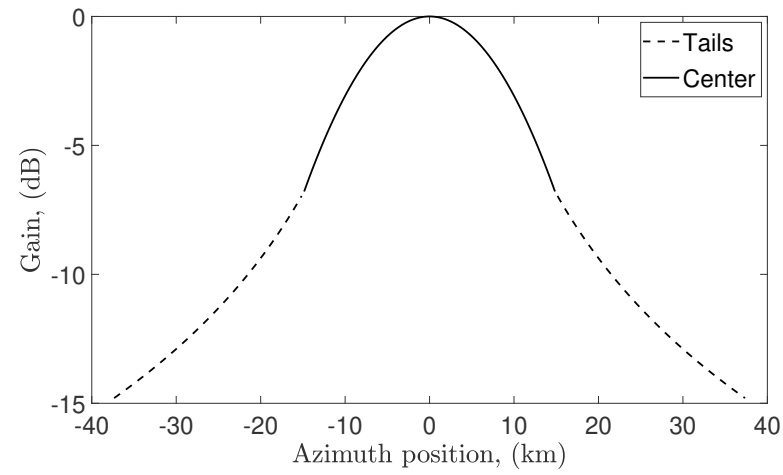

Fig. 4: One-way beam gain in azimuth direction: solid line the Gaussian centre, dash dotted line the inverse square tails.

It is worth noting that an increase in the transmitted power or in the integration time does not give a proportional increase in the Signal to Noise Ratio.

\section{Method sub-Models}

The method has a modular structure. This allows starting from crude sub-models and then replacing a specific one with a more accurate sub-model if it is needed.

\section{A. Backscatter coefficient}

The backscatter coefficient [18] is a function of landcover, season, wavelength, incidence angle and polarisation:

$$
\sigma_{i}^{0}=f_{6}\left(\Lambda_{i}, \lambda, \theta_{i}, P O L\right)
$$

\section{B. Beam gain}

The beam (one-way, power) is assumed to have a Gaussian shape for the central part and an inverse square shape for the tails, as shown in Fig. 4 and in the following equation:

$$
G_{i}=\left\{\begin{array}{cc}
\exp \left(-\left(\frac{y_{i}}{\sigma_{B}}\right)^{2}\right) & \text { for }|y| \leq \sigma_{B}, \\
\frac{1}{e} \cdot\left(\frac{\sigma_{B}}{y_{i}}\right)^{2} & \text { for }|y| \geq \sigma_{B} .
\end{array}\right.
$$

where $\sigma_{B}$ is given by the antenna characteristics.

\section{Target displacement standard deviation}

We decided to build a physics based clutter model. While the clutter model available in literature [13]-[15] model the signal received from the clutter, we try to model the movement that causes the clutter. Thus we use the crop displacement (wheat in this case). Depending on the wavelength we use we can have three contribution to the target signal: one from the top of the vegetation, one from the stocks and one from the soil. Having the data for the top of the surface movement [16], we start modelling this contribution, keeping in mind that further work is needed to achieve a complete model. 


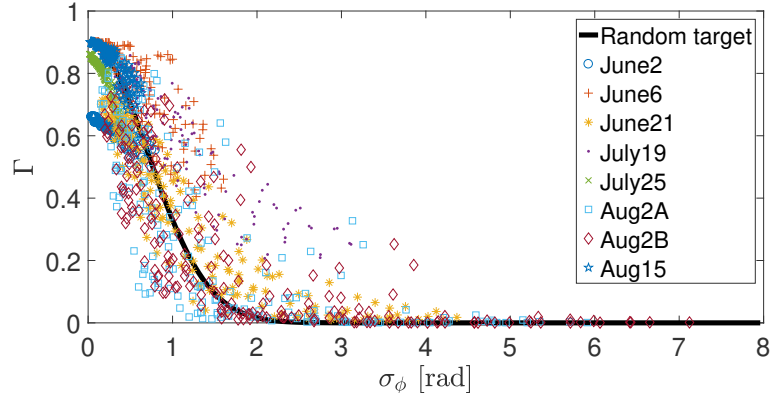

Fig. 5: Coherent power related to the $\sigma_{\phi}, \lambda=10 \mathrm{~cm}$, $v_{y}=10 \mathrm{~m} / \mathrm{s}, 20^{\circ}<\theta<70^{\circ}, 0^{\circ} \leq \psi<360^{\circ}$. The scattered points are from the wheat data, the line is the random phase target.

Our model relates the target displacement standard deviation to the mean windspeed, the landcover and the season. It is the two line fit shown in Fig. 2 and we can express it with the following equation:

$$
\sigma_{i}^{r}=f_{7}\left(W_{i}, \Lambda_{i}\right)
$$

\section{Target phase standard deviation}

The target phase standard deviation $\left(\sigma_{i}^{\phi}\right)$ of the cell $i$ is proportional to the target displacement standard deviation, as shown in the following equation:

$$
\sigma_{i}^{\phi}=\frac{4 \pi}{\lambda} \cdot \sigma_{i}^{r}
$$

\section{E. Coherent power}

The coherent power is computed using the coherent power function, together with its functional dependencies, illustrated in the following:

$$
\Gamma_{i}=f_{4}=\Gamma_{0} \cdot \exp \left(-\left(\frac{\sigma_{i}^{\phi}}{\sigma_{0}}\right)^{2}\right)
$$

where the two constants $\left(\Gamma_{0}=0.9096, \sigma_{0}=0.997 \mathrm{rad}\right)$ allow to fit the coherent power function of the wheat in summer. $\Gamma_{0}$ is dimensionless and $\sigma_{0}$ has dimension of radians.

Figure 5 shows the power in the central peak in the different days and for different geometry conditions.

\section{F. Incoherent power}

A possible function $f_{5}$ could be an exponential decay, like

$$
P D F(y)=\omega(y)=K \cdot\left(\frac{y}{\rho_{y}}\right)^{\alpha} \quad \text { for }|y|>\rho_{y}
$$

The constant $\alpha$ is dimensionless and $K$ has dimension of $\mathrm{m}^{-1}$ as the PDF.

$$
\Omega_{j}^{i}=\int_{\left|y_{i}-y_{j}\right|-\rho_{y}}^{\left|y_{i}-y_{j}\right|+\rho_{y}} \omega d y
$$

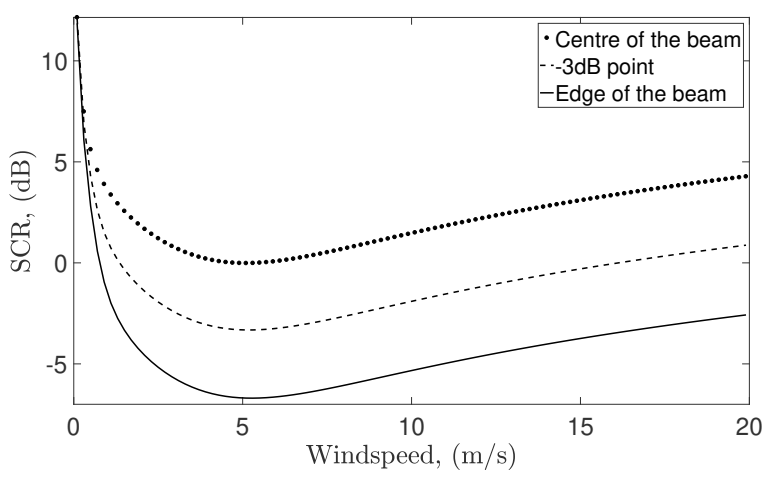

Fig. 6: SCR simulated with a triangular clutter distribution: $\lambda=3 \mathrm{~cm}, v_{y}=3.5 \mathrm{~m} / \mathrm{s}, R_{S L}=38500 \mathrm{~km}, \rho_{y}=330 \mathrm{~m}$, landcover: wheat, season: summer.

As stated in Eq.(23), the incoherent power PDF is a function of the target phase standard deviation, the wavelength, the target coherence time and the azimuth speed. Thus, the two parameters, $\alpha$ and $K$, are function of those variables.

\section{CONCLUSIONS AND FUTURE WORK}

In this paper we briefly discussed the Geosynchronous SAR mission concepts and the available land clutter literature; as a consequence we identified the need for a new clutter model in order to complete the performance estimation method. This method allows the estimation of the performance of a SAR system on a real landscape and in realistic weather conditions.

Preliminary results assuming a triangular (Fig. 6) and a rectangular (Fig. 7) clutter shape show the influence of the clutter shape. In particular, it is worth noting that over a certain mean windspeed, around 5 meters per second, the clutter is pushed outside the image and thus we have an increase of the Signal to Clutter Ratio. The orbit assumed in Fig. 6 and Fig. 7 and is the typical one of the GeoSTARe [3] mission concept.

Further work is needed to complete the short vegetation clutter model. The incoherent power model requires finding the two parameters $(\alpha$ and $K$ ) that are related. Actually there is only one independent parameter.

Further work is needed to complete the method: we have to build the sub-models for the other landcovers (e.g. forest and sea surface). This means both calibrating the coefficients of a model like the wheat one or developing a new model for each landcover.

Once completed, the Performance Estimation method could prove important not only for the low azimuth speed missions considered [3]-[5], [7]-[9] but also for other SAR missions allowing to improve the performance estimation.

\section{REFERENCES}

[1] K. Tomiyasu and J. L. Pacelli, "Synthetic Aperture Radar Imaging from an Inclined Geosynchronous Orbit," IEEE Transactions on Geoscience and Remote Sensing, vol. GE-21, no. 3, pp. 324-329, July 1983.

[2] S. N. Madsen, W. Edelstein, L. D. DiDomenico, and J. LaBrecque, "A geosynchronous synthetic aperture radar; for tectonic mapping, disaster management and measurements of vegetation and soil moisture," in International Geoscience and Remote Sensing Symposium (IGARSS), vol. 1, 2001, pp. 447-449. 


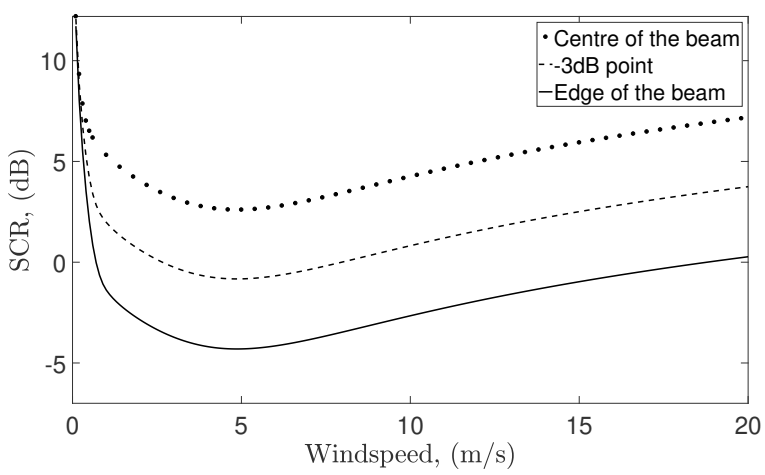

Fig. 7: SCR simulated with a rectangular clutter distribution: $\lambda=3 \mathrm{~cm}, v_{y}=3.5 \mathrm{~m} / \mathrm{s}, R_{S L}=38500 \mathrm{~km}, \rho_{y}=330 \mathrm{~m}$, landcover: wheat, season: summer.

[3] S. Hobbs, A. M. Guarnieri, G. Wadge, and D. Schulz, "GeoSTARe initial mission design," in 2014 IEEE Geoscience and Remote Sensing Symposium, July 2014, pp. 92-95.

[4] J. Ruiz-Rodon, A. Broquetas, E. Makhoul, A. M. Guarnieri, and F. Rocca, "Nearly Zero Inclination Geosynchronous SAR Mission Analysis With Long Integration Time for Earth Observation," IEEE Transactions on Geoscience and Remote Sensing, vol. 52, no. 10, pp. 6379-6391, Oct 2014.

[5] S. Hobbs, J. P. Sanchez, C. Convenevole, A. Gibbings, C. Germani, and A. M. Guarnieri, "G-class: A geosynchronous radar mission to study the diurnal water cycle," in Proceedings of the International Astronautical Congress, IAC, vol. 2018-October, 2018.

[6] C. Prati, F. Rocca, D. Giancola, and A. M. Guarnieri, "Passive geosynchronous SAR system reusing backscattered digital audio broadcasting signals," IEEE Transactions on Geoscience and Remote Sensing, vol. 36, no. 6, pp. 1973-1976, Nov 1998.

[7] A. M. Guarnieri, S. Tebaldini, F. Rocca, and A. Broquetas, "GEMINI: Geosynchronous SAR for Earth Monitoring by Interferometry and Imaging," in 2012 IEEE International Geoscience and Remote Sensing Symposium, July 2012, pp. 210-213.

[8] S. E. Hobbs, C. Convenevole, M. Gashinova, M. Cherniakov, and S. Cassidy, "Feasibility of Passive Bistatic Geosynchronous Radar Using Comsat Transmissions," in IGARSS 2018 - 2018 IEEE International Geoscience and Remote Sensing Symposium, July 2018, pp. 8026-8029.

[9] A. M. Guarnieri, A. Broquetas, A. Recchia, F. Rocca, and J. Ruiz-Rodon, "Advanced Radar Geosynchronous Observation System: ARGOS," IEEE Geoscience and Remote Sensing Letters, vol. 12, no. 7, pp. 1406-1410, July 2015.

[10] Y. Ji, Q. Zhang, Y. Zhang, and Z. Dong, "L-band geosynchronous SAR imaging degradations imposed by ionospheric irregularities," Science China Information Sciences, vol. 60, no. 6, 2017.

[11] J. R. Rodon, A. Broquetas, A. M. Guarnieri, and F. Rocca, "Geosynchronous SAR Focusing With Atmospheric Phase Screen Retrieval and Compensation," IEEE Transactions on Geoscience and Remote Sensing, vol. 51, no. 8, pp. 4397-4404, Aug 2013.

[12] S. Hobbs, C. Convenevole, A. M. Guarnieri, and G. Wadge, "Geostare system performance assessment methodology," in 2016 IEEE International Geoscience and Remote Sensing Symposium (IGARSS), July 2016, pp. 1404-1407.

[13] P. Lombardo and J. B. Billingsley, "New model for the Doppler spectrum of windblown radar ground clutter," IEEE National Radar Conference - Proceedings, pp. 142-147, 1999.

[14] J. B. Billingsley, A. Farina, F. Gini, M. V. Greco, and L. Verrazzani, "Statistical analyses of measured radar ground clutter data," IEEE Transactions on Aerospace and Electronic Systems, vol. 35, no. 2, pp. 579-593, April 1999.

[15] J. Billingsley, Low-angle Radar Land Clutter: Measurements and Empirical Models. William Andrew Pub., 2002.

[16] S. Hobbs, "Database of individual wheat plant motion in wind: Application to radar imaging of vegetation," Agricultural and Forest Meteorology, vol. 148, no. 11, pp. 1860-1868, 2008.

[17] M. I. Skolnik, Radar Handbook, ser. Electronic engineering series. McGraw-Hill, 1990.
[18] F. Ulaby and M. Dobson, Handbook of Radar Scattering Statistics for Terrain, ser. Artech House remote sensing library. Artech House, 1989. 
2019-09-27

\section{Method for estimating clutter limited geosynchronous synthetic aperture radar performance}

\section{Convenevole, Carlo}

IEEE

Convenevole C, Hobbs S. (2019) Method for estimating clutter limited geosynchronous

synthetic aperture radar performance. In: International Radar Conference: Sensing from Sea to Space, 23-27 September 2019, Toulon, France

https://dspace.lib.cranfield.ac.uk/handle/1826/15300

Downloaded from Cranfield Library Services E-Repository 